\title{
Ein pflegegeleitetes Sekundärpräventionsprogramm für Menschen nach einem akuten Koronarsyndrom aus Sicht von Gesundheitsexpertinnen und -experten in Österreich - eine qualitative Studie
}

\author{
Hong Qin ${ }^{1,2,3} \cdot$ Diana Bonderman ${ }^{3,4} \cdot$ Silvia Brunner ${ }^{1,5} \cdot$ Tamara Großbichler $^{6} \cdot$ Roza Badr Eslam $^{3} \cdot$ Hanna Mayer $^{1,7}$ \\ Eingegangen: 21. Juni 2021 / Angenommen: 30. November 2021 / Online publiziert: 6. Januar 2022 \\ (C) Der/die Autor(en) 2022
}

\section{Zusammenfassung}

Hintergrund Menschen nach akutem Koronarsyndrom weisen weiterhin ein hohes kardiovaskuläres Risiko auf. Eine pflegerische Intervention zur Verbesserung der Adhärenz von Betroffenen hinsichtlich der empfohlenen Präventionsmaßnahmen ist erforderlich. In deren Entwicklung sollen alle an der Versorgung beteiligten Personengruppen miteinbezogen werden, u. a. Gesundheitsexpertinnen und -experten.

Ziel Ziel war es, die Ausrichtung eines dem österreichischen Kontext angepassten pflegegeleiteten Sekundärpräventionsprogramms für Menschen nach akutem Koronarsyndrom aus Sicht von Gesundheitsexpertinnen und -experten zu generieren.

Methodik Die Entwicklung dieses Programms stützt sich methodisch auf das ,intervention mapping“. Um die Inhalte der ersten 3 Schritte des Intervention mapping zu erarbeiten, wurden 2 Fokusgruppeninterviews und 13 Einzelinterviews mit 27 Gesundheitsexpertinnen und -experten durchgeführt. Die Datenanalyse erfolgte anhand der thematischen Analyse nach Braun und Clarke.

Ergebnisse Die Ausrichtung des Programms bezieht sich auf die Bereiche ,personenzentrierter Ansatz“, „Akutkrankenhaus als Initialpunkt“ und ,persönliches Gespräch als zentrale Vermittlungsform“. Die erforderlichen strukturellen und personellen Rahmenbedingungen umfassen „Prioritätensetzung für die Patientenedukation im Akutkrankenhaus“, „Einrichtung einer Anlaufstelle“, „interdisziplinäre Zusammenarbeit“ und „gesetzliche Bestimmungen für die Patienteninformation“ sowie die Schaffung der Position einer „cardiac rehabilitation nurse“, welche speziell ausgebildetes Pflegepersonal übernehmen könnte. Ein vertieftes Krankheitsverständnis und ein reduzierter Angstzustand sollen die angestrebten Ergebnisse des Programms sein.

Schlussfolgerung Das zu entwickelnde Programm kann dazu dienen, die nichtmedikamentöse Sekundärprävention bei den Betroffenen im Akutkrankenhaus zu initiieren und nach der Entlassung fortzuführen. Als Rahmenbedingungen müssen eine Anlaufstelle und die Funktion einer Cardiac rehabilitation nurse geschaffen werden. Somit kann diese spezialisierte Pflegeperson die Betroffenen in der Übergangsphase begleiten, während sie die Patientenedukation unter dem personenzentrierten Ansatz durchführt.

Schlüsselwörter Myokardinfarkt · Kardiovaskuläre Pflege · Advanced Nursing Practice · Patientenedukation · Experteninterviews

Hong Qin

a9905941@unet.univie.ac.at

1 Institut für Pflegewissenschaft, Universität Wien, Alser Straße 23/Top 12+22, 1080 Wien, Österreich

2 Universitätsklinikum AKH Wien, Wiener Gesundheitsverbund, Währinger Gürtel 18-20, 1080 Wien, Österreich

3 Klinische Abteilung für Kardiologie, Universitätsklinik für Innere Medizin II, Medizinische Universität Wien, Währinger Gürtel 18-20, 1090 Wien, Österreich
4 Klinik Favoriten, Wiener Gesundheitsverbund, Wien, Österreich

5 Stadtspital Waid und Triemli, Zürich, Schweiz

6 Department Angewandte Pflegewissenschaft, FH Campus Wien, Wien, Österreich

7 Department für Allgemeine Gesundheitsstudien, Fachbereich Pflegewissenschaft - Schwerpunkt Person Centred Care \& Dementia Care Research, Karl Landsteiner Privatuniversität für Gesundheitswissenschaften, Krems an der Donau, Österreich 


\section{A nurse-led secondary prevention program for people after acute coronary syndrome from healthcare professionals' perspectives in Austria-A qualitative study}

\section{Abstract}

Background People following acute coronary syndrome are still at the highest cardiovascular risk. A nursing intervention to improve patient adherence to recommended prevention measures is required. All groups of people involved in care should participate in developing the intervention, including healthcare professionals.

Aim The aim was to generate guidance of a nurse-led secondary prevention program adjusted in the Austrian context for patients from healthcare professionals' perspectives.

Methods The development of this program is based on intervention mapping. To specify the content of the first three steps of intervention mapping, we conducted 2 focus group interviews and 13 individual interviews with 27 healthcare professionals and applied thematic analysis.

Results The guidance of the program refers to a person-centered approach, acute hospital as the initial point and personal conversation as the central mediation form. The necessary structural conditions for the program are prioritization of patient education in the hospital, establishment of a point of contact, interdisciplinary collaboration and legal provisions for patient information. In addition, a position of a cardiac rehabilitation nurse should be created, who can take over from the specially trained nurses. Understanding of the disease and reduced anxiety should be envisaged outcomes.

Conclusion The program to be developed can contribute to the initiation of nonpharmacological secondary prevention among patients in hospital and its continuation after discharge. As a prerequisite, a point of contact and a cardiac rehabilitation nurse must be established. Thus, this specialized nurse can accompany patients in the transition phase while performing patient education under the person-centered approach.

Keywords Myocardial Infarction $\cdot$ Cardiovascular care $\cdot$ Advanced nursing practice $\cdot$ Patient education $\cdot$ Expert interviews

\section{Einleitung}

Die koronare Herzkrankheit (KHK) ist weltweit die häufigste Todesursache (WHO 2020) und verursachte im Jahr 2019 13.336 Sterbefälle in Österreich (Statistik Austria 2019). Das akute Koronarsyndrom (ACS) bezeichnet die lebensbedrohlichen Phasen der KHK (Collet et al. 2020). Die Akutbehandlung bei ACS-Betroffenen umfasst die Myokardrevaskularisation, wobei diese die Risikofaktoren der KHK nicht beseitigt. Aus diesem Grund haben die Betroffenen weiterhin ein hohes kardiovaskuläres Risiko, wenn sie die notwendigen Präventionsmaßnahmen in ihrem Alltag nicht umsetzen (Pocock et al. 2020). Studien belegen, dass die Adhärenz von Betroffenen hinsichtlich der empfohlenen Lebensstilmodifikation gering ist und die Wiederaufnahmerate im ersten Jahr nach dem ACS-Ereignis bei 16,2\% liegt (Hambraeus et al. 2016). Zusätzlich nehmen nur 17,0\% der Betroffenen tatsächlich an einer kardiologischen Rehabilitation teil (Kotseva et al. 2013). Daher soll eine adäquate Sekundärprävention die Myokardrevaskularisation ergänzen und vor der Krankenhausentlassung beginnen. Die Sekundärprävention umfasst laut Guidelines der European Society of Cardiology (ESC) das biomedizinische Risikomanagement, die Pharmakotherapie, die Einschätzung der Risikofaktoren bezüglich des Lebensstils und der psychologischen Faktoren und die Unterstützung bei der Initiierung und Aufrechterhaltung der Lebensstilmodifikation (Piepoli et al. 2016). Diese wird in Form einer kardiovaskulären Re- habilitation bei den ACS-Betroffenen angeboten und wird traditionell in 3 Phasen (Mobilisation im Krankenhaus, ambulante oder stationäre Rehabilitation und langfristige Intervention) unterteilt (Dalal et al. 2015). Dabei sollen die Betroffenen v.a. in der Phase kurz nach dem ACS kontinuierlich durch die kardiologische Rehabilitation begleitet werden, um ein wiederkehrendes kardiales Ereignis zu verhindern. $\mathrm{Zu}$ achten ist auf potenzielle Versorgungslücken, die aus unterschiedlichen Gründen entstehen (Biga 2021). In diesem Zusammenhang legen die Guidelines die Initiierung der nichtmedikamentösen Sekundärprävention durch die Patientenedukation vor der Krankenhausentlassung ausdrücklich nahe (Piepoli et al. 2016).

Auf den kardiologischen Stationen des Universitätsklinikums AKH Wien findet zurzeit noch keine strukturierte individuelle Patientenedukation bezüglich der nichtmedikamentösen Sekundärprävention statt. In Österreich müssen die ACS-Betroffenen nach der Entlassung aus dem Akutkrankenhaus entweder rund 30 Tage auf einen stationären Rehabilitationsaufenthalt warten (Großschädl et al. 2014) oder sie nehmen diesen gar nicht in Anspruch (Sulzgruber et al. 2019). Ausgehend von der aufgezeigten Versorgungsituation zeigt sich eine Lücke im Bereich der Sekundärprävention in der Übergangsphase vom Akutkrankenhaus zu den Rehabilitationszentren bzw. im Rahmen der Primärversorgung. Daher ist es notwendig, ein evidenzbasiertes, an den österreichischen Kontext angepasstes pflegegeleitetes Sekundärpräventionsprogramm (pSPP) für Men- 
schen nach ACS zu entwickeln, um eine Brücke zwischen Akutkrankenhaus und Rehabilitationszentren oder Primärversorgung zu schlagen.

Auf Grundlage dieser Ausführungen setzt ein gegenwärtig laufendes Dissertationsprojekt das Ziel, ein dem österreichischen Kontext angepasstes pSPP für Menschen nach ACS zu entwickeln. Angesichts des Zieles der Sekundärprävention besteht ein pSPP aus mehreren interagierenden Komponenten: Es muss auf die Präferenzen der Betroffenen und ihrer Familienangehörigen zugeschnitten sein, damit die Präventionsmaßnahmen von den Betroffenen besser angenommen werden und in ihren Alltag integriert werden können. Weiters muss ein pSPP auch vom interprofessionellen Team getragen werden, damit es erfolgreich umgesetzt werden kann. In diesem Sinne kann das zu entwickelnde Programm als eine komplexe Intervention bezeichnet werden (Craig et al. 2013). Um dieser Komplexität gerecht zu werden, stützt sich die Entwicklung des pSPP für Menschen nach ACS methodisch auf das ,,intervention mapping“ (IM; Bartholomew Eldredge et al. 2016).

Dies wird von 3 Perspektiven, nämlich der Anwendung von Theorie und Evidenz, dem Ansatz des sozial-ökologischen Modells und der Involvierung aller Beteiligten am Entwicklungsprozess, unterstützt. Dementsprechend wurde in einem ersten Teil des Dissertationsprojektes die Sichtweise der Patientinnen und Patienten sowie deren Ehepartner/-innen bezüglich der Präventionsmaßnahmen erhoben. Bei der vorliegenden zweiten Studie wurde der Fokus auf die Sichtweise von Gesundheitsexpertinnen und -experten gelegt.

\section{Zielsetzung und Fragestellung}

Ziel dieser Studie war es, unter Berücksichtigung der Anforderungen des IM die Ausrichtung eines dem österreichischen Kontext angepassten pSPP für Menschen nach ACS aus Sicht von Gesundheitsexpertinnen und -experten zu generieren.

Daraus leiten sich die folgenden Forschungsfragen ab:

Welche Ausrichtung soll ein dem österreichischen Kontext angepasstes pSPP für Menschen nach ACS vom Akutkrankenhaus zur Primärversorgung aus Sicht von Gesundheitsexpertinnen und -experten haben?

Welche strukturellen und personellen Rahmenbedingungen sind für das pSPP notwendig? Welche Outcomes können damit bewirkt werden?

\section{Methodik}

Für die Entwicklung des pSPP wird das IM herangezogen. Es beschreibt 6 Schritte mit detaillierten Maßnahmen
(Abb. 1). Das Resultat der Schritte 1 bis 3 ist die Darstellung des Interventionsentwurfes. Da der Fokus der vorliegenden Studie auf der Entwicklung des pSPP liegt, wird auf die Schritte 1 bis 3 des IM näher eingegangen.

Im ersten Schritt des IM wird eine Problemanalyse durch alle beteiligten Stakeholder - Adressatenkreis, Expertinnen/Experten und Forschende - durchgeführt. Der Zweck besteht darin, den Kontext und die Rahmenbedingungen des Programms zu beschreiben und Ziele des Programms festzulegen. Im zweiten Schritt erfolgt die Festlegung der angestrebten Ergebnisse des Programms in Bezug auf das Verhalten des Adressatenkreises und auf die Umwelt. Zudem sollen die Beauftragten für die Implementierung des Programms identifiziert werden. Der dritte Schritt umfasst die Darstellung des Logikmodells des Programmentwurfs und zielt auf die Festlegung der theoretischen Hintergründe zur Verhaltensänderung und der Implementierungsstrategien sowie auf die Ideengenerierung für die Programmthemen ab (Bartholomew Eldredge et al. 2016).

Um die Inhalte der ersten 3 Schritte des IM zu erarbeiten, ist u.a. die Sichtweise von Gesundheitsexpertinnen und -experten in dem entsprechenden Feld von Bedeutung (Bartholomew Eldredge et al. 2016). Um diese zu erfassen, wurde auf die Methode des Experteninterviews zurückgegriffen. Experteninterviews ermöglichen durch Darstellung und Reflexion der Realität, detaillierte Auskünfte über die Problemstrukturierung zu erhalten. Gleichzeitig fließt damit das Kontextwissen, das für eine Programmimplementierung notwendig ist, in die Ergebnisse ein (Mayer 2019; Meuser und Nagel 2009). Weiters wurde die Methode des Fokusgruppeninterviews als zentrale Erhebungsmethode gewählt, da mit dieser die Meinungen der Einzelnen in der Gruppe zu einem Thema erhoben werden sowie ein breiteres Verständnis und eine tiefere Einsicht durch die Anregungen in der Gruppe diskursiv erlangt werden können (Mayer 2019). Da hier ein möglichst breites Spektrum an Gesundheitsexpertinnen und -experten hinzugezogen werden sollte, entschied man sich für eine pragmatische Lösung für diejenigen, die aus organisatorischen bzw. terminlichen Gründen nicht an einem Fokusgruppeninterview teilnehmen konnten. Mit diesen Teilnehmenden wurden Einzelinterviews geführt.

Als Stimulus für die Fokusgruppeninterviews und Einzelinterviews diente eine Fallvignette. Ihr Einsatz im Gebiet der professionsanalytischen Forschungen dient dazu, das implizite Wissen bzw. die impliziten Wahrnehmungs-, Beurteilungs- und Entscheidungskriterien von Expertinnen und Experten zu rekonstruieren (Schnurr 2003). In der Fallvignette wird ein exemplarischer Krankheitsverlauf von einem Patienten nach ACS beschrieben. Die Fokusgruppeninterviews sowie die Einzelinterviews wurden ausgehend von diesem Stimulus auf Basis von offenen Diskussionsfragen (Froschauer und Lueger 2020) geführt. 


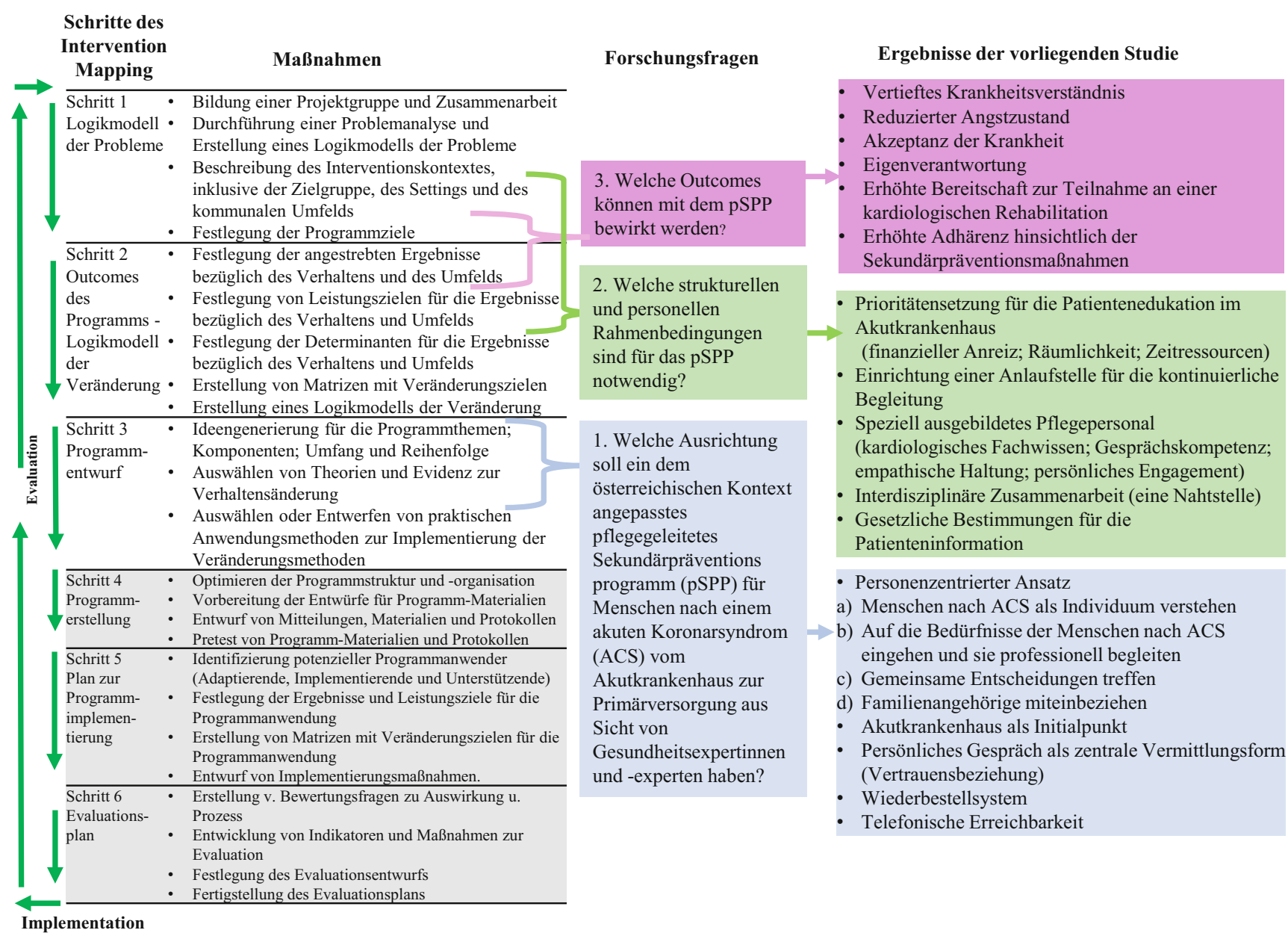

Abb. 1 Ausrichtung des pflegegeleiteten Sekundärpräventionsprogramms und der angestrebten Outcomes gemäß den Schritten des ,intervention mapping“ (Bartholomew Eldredge et al. 2016, S. 13)

\section{Teilnehmende und Setting}

Die Zielgruppe setzte sich aus Expertinnen und Experten der verschiedenen Gesundheitsberufe aus 3 Settings - Akutkrankenhäuser, stationäre oder ambulante Rehabilitationszentren und Arztpraxen der Primärversorgung für Allgemeinmedizin oder Kardiologie - zusammen, die über eine klinische Expertise bei der Betreuung von Menschen mit ACS verfügen. Alle Teilnehmenden wurden unter Berücksichtigung der Definition nach Meuser und Nagel (2009) ausgewählt. Diese verstehen unter einer Expertin oder einem Experten eine Person, die ,in irgendeiner Weise Verantwortung trägt für den Entwurf, die Ausarbeitung, die Implementierung und/oder die Kontrolle einer Problemlösung, und damit über einen privilegierten Zugang zu Informationen über Personengruppen, Soziallagen, Entscheidungsprozesse, Politikfelder usw. verfügt“ (Meuser und Nagel 2009, S. 470).

Das Forschungsteam verfolgte zwei Rekrutierungsstrategien. Erstens wurde das Projekt von der Erstautorin bei einer ärztlichen Morgenbesprechung an der kardiologischen
Abteilung des Universitätsklinikums AKH Wien präsentiert. Danach wurden die Ärztinnen und Ärzte direkt angesprochen. Mit Unterstützung der zuständigen Bereichsleitung der Pflege wurde für diplomierte Gesundheits- und Krankenpflegepersonen der kardiologischen Abteilung ein Fokusgruppeninterview organisiert. Zweitens wurden mittels Schneeballprinzips potenzielle Teilnehmende in anderen Akutkrankenhäusern, in den Rehabilitationszentren und in der Primärversorgung per E-Mail angefragt. Insgesamt wurden 29 Gesundheitsexpertinnen und -experten für die Teilnahme an der Studie angefragt, davon gaben zwei Expertinnen keine Interviews aufgrund fehlender zeitlicher Ressourcen.

\section{Datenerhebung}

Im Vorfeld der Datenerhebung wurden die Fallvignette und die „Experteninformationen und Einwilligungserklärung zur Teilnahme an der Studie“ den Expertinnen und Experten nach der mündlichen Zusage zur Teilnahme an der Studie zugeschickt. Somit konnten sie sich für die 
Tab. 1 Soziodemografische Daten der Gesundheitsexpertinnen und -experten

\begin{tabular}{|c|c|c|c|c|c|}
\hline Interview-Nr. & Beruf der Teilnehmenden & Geschlecht & Alter & Berufsjahr & Setting \\
\hline \multirow[t]{7}{*}{$\overline{\text { FI1 }}$} & DGKP & Männlich & 58 Jahre & 39 Jahre & Krankenhaus \\
\hline & DGKP & Männlich & 54 Jahre & 20 Jahre & \\
\hline & DKGP & Männlich & 50 Jahre & 19 Jahre & \\
\hline & DGKP & Männlich & 35 Jahre & 12 Jahre & \\
\hline & DGKP & Weiblich & 52 Jahre & 31 Jahre & \\
\hline & DGKP & Weiblich & 47 Jahre & 26 Jahre & \\
\hline & DGKP & Weiblich & 43 Jahre & 22 Jahre & \\
\hline \multirow[t]{7}{*}{ FI2 } & $\mathrm{AM}$ & Männlich & 38 Jahre & 9 Jahre & Stationäres RZ \\
\hline & Kardiologin & Weiblich & 54 Jahre & 30 Jahre & \\
\hline & Diätologin & Weiblich & 53 Jahre & 33 Jahre & \\
\hline & DGKP & Weiblich & 45 Jahre & 25 Jahre & \\
\hline & Physiotherapeutin & Weiblich & 37 Jahre & 14 Jahre & \\
\hline & Psychologin & Weiblich & 29 Jahre & 2 Jahre & \\
\hline & DGKP & Weiblich & 49 Jahre & 24 Jahre & \\
\hline EI1 & Kardiologe & Männlich & 45 Jahre & 20 Jahre & Ambulantes RZ \\
\hline EI2 & $\mathrm{AM}$ & Männlich & 70 Jahre & 40 Jahre & Primärversorgung \\
\hline EI3 & Internist & Männlich & 54 Jahre & 32 Jahre & Primärversorgung \\
\hline EI4 & Kardiologin & Weiblich & 55 Jahre & 29 Jahre & Primärversorgung \\
\hline EI5 & Internist & Männlich & 58 Jahre & 18 Jahre & Krankenhaus \\
\hline EI6 & Soziologe & Männlich & 60 Jahre & 35 Jahre & Krankenhaus \\
\hline EI7 & Kardiologin & Weiblich & 53 Jahre & 29 Jahre & Krankenhaus \\
\hline EI8 & Kardiologe & Männlich & 56 Jahre & 30 Jahre & Krankenhaus \\
\hline EI9 & Kardiologe & Männlich & 46 Jahre & 16 Jahre & Krankenhaus \\
\hline EI10 & Kardiologin & Weiblich & 43 Jahre & 14 Jahre & Krankenhaus \\
\hline EI11 & DGKP & Weiblich & 54 Jahre & 32 Jahre & Krankenhaus \\
\hline EI12 & DGKP & Weiblich & 48 Jahre & 30 Jahre & Stationäres RZ \\
\hline EI13 & Kardiologin & Weiblich & 53 Jahre & 18 Jahre & Krankenhaus \\
\hline
\end{tabular}

AM Allgemeinmediziner, DGKP diplomierte Gesundheits- und Krankenpflegerin oder diplomierter Gesundheits- und Krankenpfleger, EI Einzelinterview, FI Fokusgruppeninterview, $R Z$ Rehabilitationszentrum

Interviews mit dem Fokus auf das Forschungsthema vorbereiten. Die Fallvignette und die Interviewfragen wurden zuvor durch eine Expertin und einen Experten einem Pretest unterzogen und insbesondere auf Verständlichkeit und Vollständigkeit überprüft.

Die Datenerhebung fand im Zeitraum November 2018 bis Oktober 2019 statt. Die Teilnehmenden gaben vorab ihre schriftlich informierte Zustimmung und ihre persönlichen Daten (Geschlecht, Alter, Dauer der Berufserfahrung und berufliches Setting) bekannt. Sowohl die Fokusgruppeninterviews als auch die Einzelinterviews wurden digital aufgezeichnet und im Anschluss wortwörtlich transkribiert und pseudonymisiert. Die durchschnittliche Dauer beträgt bei den Fokusgruppeninterviews 67 min und bei den Einzelinterviews $37 \mathrm{~min}$.

\section{Datenanalyse}

Die Daten wurden mittel der Software MAXQDA 2020 von VERBI Software (Consult-Sozialforschung GmbH,
Berlin, Deutschland) organisiert. Die Datenanalyse der Fokusgruppeninterviews und Einzelinterviews erfolgte anhand der thematischen Analyse mit 6 Schritten nach Braun und Clarke (2006). Mit dieser Methode ist es zum einen möglich, induktiv vorzugehen, d.h., die Daten Zeile für Zeile zu kodieren und die textnahen Codes zu potenziellen Themen zu bündeln, und zum anderen, die Codes oder die potenziellen Themen den Schritten und Maßnahmen des IM zuzuordnen. Dazu wurden die Transkripte wiederholt gelesen, von drei Forschenden unabhängig kodiert, Codes gebündelt und übergeordnete Themen formuliert. Schließlich wurden diese Themen den Forschungsfragen zugeordnet.

\section{Ethische Überlegungen}

Die Ethikkommission der Medizinischen Universität Wien stimmte der Untersuchung zu (EK Nr. 1811/2018). Die schriftlichen Einverständniserklärungen der Teilnehmenden wurden vor Beginn der Interviews eingeholt. 


\section{Ergebnisse}

Von November 2018 bis Oktober 2019 nahmen 27 Gesundheitsexpertinnen und -experten, davon 15 Frauen und 12 Männer, im Alter von 29 bis 70 Jahren an der Studie teil. Insgesamt konnten 2 Fokusgruppeninterviews und 13 Einzelinterviews von der Erstautorin realisiert werden. Die Teilnehmenden waren in 3 Akutkrankenhäusern, 3 Rehabilitationszentren und Arztpraxen der Primärversorgung tätig. Die durchschnittliche Berufserfahrung der Teilnehmenden betrug 24,0 Jahre (Tab. 1).

In den folgenden Abschnitten werden die Ergebnisse aus den Interviews mit Gesundheitsexpertinnen und -experten entlang den 3 Forschungsfragen vorgestellt. Die in der Auswertung zugeordneten Codes werden als zusätzliche Inhalte des pSPP fett hervorgehoben. Zur besseren Nachvollziehbarkeit und Übersichtlichkeit werden in Abb. 1 die Ergebnisse parallel zu den Schritten des IM dargestellt und den Forschungsfragen zugeordnet.

\section{Welche Ausrichtung soll das pSPP haben?}

Das zu entwickelnde pSPP soll einen personenzentrierten Ansatz verfolgen und im Akutkrankenhaus beginnen.

\section{Personenzentrierter Ansatz}

In diesem Zusammenhang sind folgende 4 Strategien wesentlich: 1) Menschen nach ACS als Individuum verstehen, 2) auf die Bedürfnisse der Menschen nach ACS eingehen und sie professionell begleiten, 3) gemeinsame Entscheidungen treffen und 4) Familienangehörige miteinbeziehen.

Die Mehrzahl der Patientinnen und Patienten befindet sich nach dem ACS-Ereignis in einem Ausnahmezustand. Einerseits sind die meisten Betroffenen sehr froh, dass sie dieses Ereignis überlebt haben. Andererseits sind sie überfordert. „Da brauchen wir wirklich gutes Verständnis, dass nicht alles rüberkommt, und da brauchen wir Repetition, da brauchen wir verschiedene Kommunikationskanäle, angstfreie Umgebung " (EI3:176-178).

Bei den ACS-Betroffenen, die gar keine Beschwerden haben und immer sportlich sind, steigen Angst und Ungewissheit, weil das zukünftige Leben außer Kontrolle geraten könnte. Vor diesem Hintergrund empfahlen die Teilnehmenden, die psychischen Probleme der Betroffenen zu beachten, indem sie als Individuum verstanden werden und eine Vertrauensbeziehung mit ihnen durch persönliche Gespräche aufgebaut wird. „Es geht einfach darum, dass er eben seine Sorgen, Ängste, die jetzt dann langsam eben zum Tragen kommen, dass er die irgendwo formulieren kann. [...] es geht ganz besonders einfach auch um das Reden" (EI7:48-50).
Die Reaktionen der Betroffenen auf das ACS-Ereignis sind sehr unterschiedlich. Für die meisten bedeutet das ACS eine existenzielle Bedrohung im Leben, weshalb sie z.B. sofort mit dem Rauchen aufhören. Andere jedoch erwecken den Eindruck, sich geheilt zu fühlen, z. B. wollen entlassen werden, sobald sie nach der invasiveren Behandlung aufstehen dürfen. Während manche Betroffene über ihren Zustand Bescheid wissen wollen, möchten andere nichts davon hören. Zusätzlich sind die Bedürfnisse der Betroffenen aufgrund ihrer kulturellen und religiösen Hintergründe sowie ihrer sozialen Umgebung sehr unterschiedlich, wie das folgende Zitat zeigt: ,... der ist einmal froh, dass er überlebt hat, und überlegt sich, wie geht das jetzt weiter? Bin ich jetzt mein Leben lang krank? Kann ich so arbeiten, wie ich bisher gearbeitet habe? Muss ich in meinem Familienleben etwas umstellen?" (EII:30-33). Daher soll bei der Informationsvermittlung auf die individuellen Bedürfnisse eingegangen werden.

Die professionelle Begleitung muss bei der Förderung der Selbstmanagementfähigkeit gewährleistet sein: „Wenn ich ihm sage, er soll Selbstmanagement betreiben, Blutdruck messen, Puls messen, dann sage ich ihm Normalwerte und dann ist er dauernd draußen, wo geht er jetzt hin einen Monat?" (FI1:692-694). Genauso wie bei der Lebensstiländerung können die Betroffenen durch die professionelle Begleitung einerseits adressatengerechte Informationen erhalten, um persönliche Entscheidungen zu treffen, andererseits alltagstaugliche Strategien entwickeln und konsequent umsetzen.

Entscheidungen über Präventionsmaßnahmen und das anzustrebende Ziel sollen mit den Betroffenen gemeinsam getroffen, in einer verbindlichen Form schriftlich festgelegt und individuell gestaltet werden. Den Betroffenen wird dadurch bewusst, dass sie für die Durchführung selbst verantwortlich sind.

Der Einbezug der Familienangehörigen in das Programm ist essenziell. Wenn die Familienangehörigen dieselben Informationen bekommen wie die Patientinnen und Patienten, können sie die betroffene Person bei der Umsetzung und der Aufrechterhaltung der Präventionsmaßnahmen im Alltag unterstützen.

\section{Akutkrankenhaus als Initialpunkt}

Die ACS-Betroffenen sollen im Akutkrankenhaus rechtzeitig über die KHK informiert werden. Die Patientenedukation soll dazu dienen, „dem Patienten bewusst zu machen, was hat denn eigentlich zu seinem Infarkt geführt? Und diese persönliche Sicht des Patienten zu erfragen, sonst sehen auch viele Patienten nicht wirklich einen Sinn, etwas zu verändern" (EI13:53-55). Die Teilnehmenden sahen die Notwendigkeit, dass „,die Akutversorgung die Aufgabe kriegen müsste" (EI6:79). Idealerweise sollte die Kardiologin oder 
der Kardiologe gleich nach der Herzkatheteruntersuchung mit den Betroffenen kurz sprechen - ,Ich habe Ihnen zwar jetzt das Gefäß eröffnet, aber jetzt beginnt ein Weg für Sie! “ (EI13:307-308) - und auf das Programm hinweisen. Vor der Entlassung können die Betroffenen durch umfassende Informationen mittels des pSPP ihre Krankheitswahrnehmung erhöhen und sich mehr Wissen über die Präventionsmaßnahmen aneignen. Nach der Entlassung werden sie durch ein Wiederbestellsystem vom Gesundheitspersonal aktiv kontaktiert und kontinuierlich begleitet.

\section{Persönliches Gespräch als zentrale Vermittlungsform}

Durch ein persönliches Gespräch kann eine Vertrauensbeziehung zwischen den Betroffenen und dem Gesundheitspersonal hergestellt werden. Auf dieser Basis sind ACSBetroffene offen für die Informationen und auch bereit, an diesem Programm teilzunehmen.

Es setzt voraus, [...] dass die betreuende Person eine große Vertrauensperson ist. Und wenn die permanent wechselt, dann ist zwar vielleicht auch die Therapie dieselbe, aber es funktioniert trotzdem nicht mehr so gut. (EI3:250-255)

Als weitere Vermittlungsformen nannten die Teilnehmenden eine interaktive Website, telefonische Beratung und schriftliche Informationen als Ergänzungen zum persönlichen Kontakt. „Also zuerst ein erstes Gespräch, und dann Verweisen auf eine Internetseite, verlässliche! " (EI6:127). Außerdem unterstrichen sie die Notwendigkeit der telefonischen Erreichbarkeit.

\section{Welche strukturellen und personellen Rahmenbedingungen sind für das PSPP notwendig?}

Die Interviewauswertung zeigte, dass folgende Voraussetzungen für die Implementierung und die Durchführung des pSPP geschaffen werden müssen.

\section{Prioritätensetzung für die Patientenedukation im Akutkrankenhaus}

Von den Teilnehmenden wurde kritisiert, dass die Patientenedukation in der aktuellen Situation nicht als gesundheitspolitische und organisatorische Priorität gesehen wird. Sie betonten die Relevanz einer finanziellen Lösung seitens Bund, Länder und Sozialversicherungen, besonders einen finanziellen Anreiz für die Patientenedukation. „Die Organisationen selber bewerten das hoch und sagen, das ist wichtig, da wird Personal dafür zur Verfügung gestellt. Es ist Aufgabe unserer Einrichtung, Patienten gut zu informieren, anzuleiten" (EI6:28-30).
Um die Lebensumstände der Betroffenen kennenzulernen und ihre Ressourcen und Bedürfnisse zu eruieren, werden sowohl Räumlichkeiten als auch mehr Zeitressourcen für eine individuelle Beratung benötigt.

\section{[...] dass die nichtmedikamentöse Sekundärprävention sehr aufwendig ist, und das kann ich nicht zwischen zwei Patienten oder zwischen Tür und Angel machen. Sondern da muss ich den Patienten kennen, ich muss seine Biografie kennen, seine Lebensumstände und da- rauf eingehen können. Und das erfordert Zeit und Ru- he. (FI1:395-399)}

\section{Einrichtung einer Anlaufstelle}

Zurzeit gibt es weder eine Struktur noch die Zeit- und Personalressourcen im Akutkrankenhaus und in Arztpraxen in der Primärversorgung für Allgemeinmedizin oder Kardiologie, um die nichtmedikamentöse Sekundärprävention bei Menschen nach ACS rechtzeitig und kontinuierlich durchzuführen. Deshalb brachten die Teilnehmenden sehr konkret zur Sprache, dass ,eine Anlaufstelle für diese Patienten [...] eingerichtet werden soll" (EI7:164-165). Diese Anlaufstelle dient dazu, die Unsicherheiten der Betroffenen aufzufangen, ihre Fragen zu beantworten zu lassen, und das pSPP anzubieten. In der Erwägung, wo die Anlaufstelle eingerichtet werden soll, damit die Betroffenen kontinuierlich begleitet werden, zeigten sich in den Interviews verschiedene Überlegungen, wie folgendes Zitat verdeutlicht:

Wenn ich nach pflegerischen Perspektiven suche, dann geht es natürlich in die Richtung der Stationen. Aber dafür ist das Akutkrankenhaus nicht ausgerichtet. Aber dann geht es in die Richtung Patienteninformationszentrum mit einem entsprechend kompetent ausgebildeten Pflegepersonal. [...] Da kann der Patient hingehen. Was aber trotzdem fehlt, ist die Nachverfolgung, weil die Patienten zu Hause dann mehr oder weniger allein sind. Also muss man auch eine Verbindung schaffen. (FI1:407-416)

\section{Speziell ausgebildetes Pflegepersonal}

In Bezug auf personelle Rahmenbedingungen sollte die Position einer „cardiac rehabilitation (CR) nurse“ als Beauftragte für die Implementierung und die Durchführung des pSPP geschaffen werden. Die Teilnehmenden aus dem Pflegeberuf und aus anderen medizinischen Berufen teilten die Meinung, dass das speziell ausgebildete Pflegepersonal diese Position übernehmen könne.

Die Teilnehmenden aus dem Pflegeberuf meinten dazu, dass das Pflegepersonal in der Kardiologie den Betroffenen die medizinischen Informationen auf Basis ihrer klinischen 
Erfahrungen für Laiinnen und Laien verständlich erklären könne.

Aber die Teilnehmenden sahen auch die Notwendigkeit einer speziellen Ausbildung für das Pflegepersonal, um die sozialen und medizinischen Kompetenzen für ihr eigenständiges Handeln als CR nurses zu erwerben: „Sie sollten irgendwie geschult sein! " (EI4:542).

Die CR nurses sollen neben ihrem kardiologischen Fachwissen über Gesprächskompetenz verfügen und besonders motivierende Gesprächsführung kennen, weil dieser Beratungsansatz gut geeignet ist, die Betroffenen zur Lebensstiländerung zu motivieren. Hinsichtlich der Gesprächskompetenzen nannten die Teilnehmenden: zuzuhören, Fragen stellen zu lassen, Feedback von den Betroffenen einzuholen und gemeinsame Entscheidungen zu treffen. Zusätzlich sollen die CR nurses die Kommunikation mit den Betroffenen effizient gestalten, wie das folgende Zitat verdeutlicht: ,Entscheidend ist, dass wir nicht nur auf der akustischen Schiene arbeiten mit unseren Patienten. Denn sehr wenige Menschen sind rein akustisch orientiert. Die meisten Menschen sind visuell oder kinästhetisch-haptisch. Das heißt, wir müssen die Kommunikation, die wir haben, ändern “ (EI3:92-95).

Darüber hinaus wurde hervorgehoben, dass für die Gesprächsführung mit den Betroffenen empathische Haltung und persönliches Engagement erforderlich seien.

\section{Interdisziplinäre Zusammenarbeit}

Eine interdisziplinäre Zusammenarbeit sowohl in der Organisation als auch über Setting-Grenzen hinaus im Sinn einer sektorenübergreifenden Vernetzung, welche durch eine Nahtstelle ermöglicht wird, wurde als eine wichtige Voraussetzung für eine kontinuierliche Versorgung bei Menschen nach ACS gesehen. Die CR nurses könnten die Koordinationsfunktion in dieser Nahtstelle übernehmen: Einerseits seien sie als zentrale Ansprechperson für die Betroffenen da, andererseits: „Sie sollten vernetzt sein mit den Systemen, mit denen sie zusammen für den Patienten arbeiten" (EI4:553).

Zusätzlich sollte das beteiligte Gesundheitspersonal die aktuellen Versorgungsleitlinien einhalten und den Betroffenen gleiche Informationen geben, um Verwirrungen durch unterschiedliche Informationen $\mathrm{zu}$ vermeiden.

\section{Gesetzliche Bestimmungen für die Patienteninformation}

Die Teilnehmenden sahen zurzeit eine gesetzliche Lücke bezüglich der Patienteninformation durch die nichtärztlichen Gesundheitsberufe. Sie betonten die Notwendigkeit des Vorhandenseins der entsprechenden gesetzlichen Bestimmungen. Somit könnten die Patienteninformation und die Aufklärung als Dienstleistung von den anderen Gesund- heitsberufen neben den Ärztinnen/Ärzten durchgeführt und als solche finanziert werden.

\section{Welche Outcomes können mit dem pSPP bewirkt werden?}

Als die angestrebten Outcomes des pSPP wurden u. a. ,,vertieftes Krankheitsverständnis“ und „reduzierter Angstzustand" genannt.

\section{Vertieftes Krankheitsverständnis}

Vertieftes Krankheitsverständnis ist einer der entscheidenden Faktoren, die im Zusammenhang mit Adhärenz hinsichtlich der Sekundärpräventionsmaßnahmen stehen: „Wie es zu der Erkrankung gekommen ist. Die Krankheit verstehen. Warum sie Medikamente nehmen sollen. [...] Dass sie eine Idee dafür bekommen, was sie in ihrem Leben verändern möchten, und auch eine Idee, wie sie ihr Leben verändern können " (EI13:338-342).

Das erste ACS-Ereignis ist häufig der Beginn einer chronischen Erkrankung. Diese erfordert, dass Betroffene sich die empfohlenen Präventionsmaßnahmen systematisch aneignen und dann eigene Präventionsmaßnahmen in ihren Alltag integrieren, um das Fortschreiten der Erkrankung zu verlangsamen. Aufgrund der raschen Behandlung und der verkürzten Aufenthaltsdauer im Krankenhaus sind die Betroffenen meistens nicht in der Lage, sich mit der Krankheit auseinanderzusetzen, und können in der Folge auch kein Verständnis für die KHK entwickeln. Durch einen personenzentrierten Ansatz, v. a. durch die aktive Einbindung der Betroffenen in das pSPP, können sie einerseits Informationen über Chronizität und Ursachen der KHK sowie über die Notwendigkeit einer kardiologischen Rehabilitation und der Umstellung der eigenen Verhaltensweise rechtzeitig, dosiert und wiederholt erhalten. Andererseits können sie durch den persönlichen Kontakt mit CR nurses eine kontinuierliche Begleitung bekommen, die für die Entwicklung des Krankheitsverständnisses notwendig ist. Im Weiteren fördert das pSPP die Akzeptanz der Krankheit und die Eigenverantwortung für die Gesundheit. Schließlich steigern sich ihre Bereitschaft zur Teilnahme an einer kardiologischen Rehabilitation und die Adhärenz hinsichtlich der Sekundärpräventionsmaßnahmen.

\section{Reduzierter Angstzustand}

Angst ist nicht nur ein wichtiger Faktor für die Verhaltensänderung, sondern auch mit dem Risiko für wiederkehrende kardiovaskuläre Ereignisse und psychosomatische Erkrankungen verbunden. Das akute ACS-Ereignis löst bei den meisten Betroffenen Angst und Unsicherheit aus. Dadurch verfügen sie über eine eingeschränkte Aufnahmefähigkeit - 
„Weil mit Angst im Kopf lernt es sich schlecht. Diese Dinge müssen wir berücksichtigen “ (EI3:178-179). Weiters wollen die Betroffenen aufgrund der Angst vor einem wiederkehrenden Herzinfarkt nichts über die Krankheit wissen und meiden Bewegung.

\section{Diskussion}

Die Studienergebnisse zeigen, dass sich die Gesundheitsexpertinnen und -experten über das zu entwickelnde pSPP im österreichischen Kontext sehr einig sind. Die Ausrichtung des Programms bezieht sich auf die Bereiche, ,personenzentrierter Ansatz“, ,Akutkrankenhaus als Initialpunkt“ und „persönliches Gespräch als zentrale Vermittlungsform“. Die erforderlichen strukturellen und personellen Rahmenbedingungen umfassen ,Prioritätensetzung für die Patientenedukation im Akutkrankenhaus“, „Einrichtung einer Anlaufstelle“, ,interdisziplinäre Zusammenarbeit" und ,gesetzliche Bestimmungen für die Patienteninformation" sowie die Schaffung einer Position einer CR nurse, welche speziell ausgebildetes Pflegepersonal einnehmen kann. Ein vertieftes Krankheitsverständnis und ein reduzierter Angstzustand sollen die angestrebten Ergebnisse des Programms sein.

Das pSPP soll einen personenzentrierten Ansatz verfolgen und im Akutkrankenhaus beginnen. Zugleich sollen die Patienteninformationen durch persönliche Gespräche vermittelt werden. Der personenzentrierte Ansatz bezieht sich auf eine Reihe von Grundsätzen: Berücksichtigung der Überzeugungen und Werte der Betroffenen, authentisches Engagement, einfühlsame Präsenz, gemeinsame Entscheidungsfindung, ganzheitliche Pflege (McCance und McCormack 2017). Diese spiegeln sich in der vorliegenden Studie wider. Der personenzentrierte Ansatz wird auch von $\mathrm{Hu}-$ riani (2019) empfohlen, die die Sichtweise von Menschen nach ACS erforscht hat. Zudem soll sich das Programm an der Theorie des Erwachsenenlernens orientieren.

Eine Studie von Auer et al. (2008) zeigt, dass die Mortalitätsrate von Menschen nach ACS sinkt, wenn die Sekundärpräventionsmaßnahmen im Akutkrankenhaus beginnen und auf allen Ebenen nach der Entlassung aus dem Krankenhaus fortgeführt werden. Diesen Standpunkt vertraten auch die Teilnehmenden dieser Studie, zugleich hoben sie das persönliche Gespräch mit den Betroffenen hervor. In diesem Zusammenhang stellt Berra (2010) fest, dass ständiger Informationsaustausch und die Zufriedenheit mit dem Gesundheitspersonal positive Auswirkungen auf die Selbstmanagementfähigkeit der Patientinnen und Patienten hinsichtlich der Verhaltensänderung haben.

Laut Patientencharta (2006) gilt die Patientenedukation als eine Kernleistung der Krankenversorgung. Demzufolge sollen die gesundheitssystembezogenen Rahmenbedingungen, d. h. Finanzierung, Leistungszuordnung und Rollenver- teilung, geschaffen werden, um die Patientenedukation im Versorgungsalltag zu ermöglichen (BMGF 2016). Hingegen sieht die Realität anders aus, und es finden sich zahlreiche Hürden, wie unzureichende Zeit- und Arbeitskapazität, fehlende finanzielle Anreize, das auf die Akutversorgung konzentrierte Gesundheitswesen und der Mangel an präventiven Strukturen auf der Ebene des Gesundheitssystems (Clark et al. 2013). In der vorliegenden Studie betonten die Teilnehmenden, dass die Patientenedukation als eine zentrale Leistung des Akutkrankenhauses gesehen werden solle. Diese Priorisierung der Patientenedukation im Versorgungsalltag gilt als eine wesentliche Rahmenbedingung für das pSPP. Menschen nach ACS befinden sich in einem Ausnahmezustand und gehören daher zu einer vulnerablen Personengruppe. Um dieser spezifischen Vulnerabilität besser begegnen zu können, ist das Gesundheitspersonal verpflichtet, ein Verständnis für die Situation der Betroffenen zu entwickeln (Schrems 2019). Dementsprechend ist es mit viel Energie und Zeitaufwand verbunden, ihnen die Sekundärpräventionsmaßnahmen im Rahmen der Sekundärprävention zu erläutern und sie im Zuge dessen zu begleiten. In diesem Zusammenhang zeigt die Untersuchung von Flodgren et al. (2011), dass finanzielle Anreize zur Verbesserung der Versorgungsprozesses führen können. Zusätzlich wurde von den Teilnehmenden eine sektorenübergreifende Vernetzung auf Basis der interdisziplinären Zusammenarbeit als eine wesentliche Rahmenbedingung für die Implementierung des pSPP unterstrichen.

Das Gesundheitspersonal soll die Sekundärpräventionsmaßnahmen rechtzeitig bei Menschen nach ACS einleiten und sie bei der Umsetzung solcher Maßnahmen unterstützen. Dies kann nur durch eine kontinuierliche Begleitung realisiert werden (Biga 2021). In diesem Zusammenhang sahen die Teilnehmenden die Notwendigkeit, dass eine Anlaufstelle für die Betroffenen - auch im Sinne einer Nahtstelle für eine sektorenübergreifende Vernetzung - eingerichtet wird. In diesem Zusammenhang empfiehlt Huriani (2019) ebenso die Errichtung einer offiziellen Struktur, um die Durchführung einer hochwertigen Patientenedukation systematisch sicherzustellen. Wie Wienbergen et al. (2018) in ihren Untersuchungen bestätigen, erzielen die von einer solchen Einrichtung durchgeführten Interventionen effektive Ergebnisse bei Menschen nach ACS.

Aus den Ergebnissen dieser Studie geht hervor, dass die Position einer CR nurse für die Implementierung und die Durchführung der Patientenedukation geschaffen werden muss. Speziell ausgebildetes Pflegepersonal kann diese Funktion übernehmen und das pSPP leiten. Die CR nurse ist einerseits die Ansprechperson für die Betroffenen und andererseits eine Koordinatorin zwischen unterschiedlichen Versorgungssektoren. Studien belegen, dass pflegegeleitete Präventionsprogramme zur Reduktion von kardiologischen Risikofaktoren führen, indem ausgebildetes Pflege- 
personal den Patientinnen und Patienten beim Blutdruckmanagement, der Lipidsenkung, der Ernährungsumstellung und dem Aufbau der körperlichen Aktivität zur Seite steht (Snaterse et al. 2016), sowie auch die Teilnahme an einer Rehabilitation fördert (Cossette et al. 2012). Solche Programme zielen auf die Lebensführung mit der Krankheit $\mathrm{ab}$, statt auf die Behandlung der Krankheit zu fokussieren (The Joanna Briggs Institute 2010). Des Weiteren sollen die CR nurses über Gesprächskompetenz verfügen und besonders motivierende Gesprächsführung kennen und anwenden, welche sich als effektive pflegerische Intervention zur Verbesserung der Krankheitswahrnehmung bei Patientinnen und Patienten mit KHK gezeigt hat (Mülhauser et al. 2018).

In Bezug auf angestrebte Outcomes des pSPP unterstrichen die Teilnehmenden ein vertieftes Krankheitsverständnis, weil dies einer der entscheidenden Faktoren ist, die im Zusammenhang mit Adhärenz hinsichtlich Sekundärpräventionsmaßnahmen stehen. Cossette et al. (2012) und Mülhauser et al. (2018) setzen die Krankheitswahrnehmung als ein wichtiges Ergebniskriterium ihrer Interventionen ein. Dies ist damit begründet, dass die Berücksichtigung der Krankheitswahrnehmung der Betroffenen zur Förderung der Krankheitsbewältigung, Reduktion der Depression und $\mathrm{zu}$ verbesserter Lebensqualität führen kann (Alsen et al. 2010). Hinsichtlich psychischer Probleme wird berichtet, dass über $30 \%$ der ACS-Betroffenen zum Zeitpunkt des Ereignisses und auch in der Frühgenesungsphase ängstlich oder depressiv sind (Murphy et al. 2019). Solches psychologische Wohlbefinden beeinflusst sowohl die Krankheitswahrnehmung als auch die Annahme von Hilfestellung, insbesondere die Teilnahme an einer kardiologischen Rehabilitation (Dalal et al. 2015). Daher setzen Oranta et al. (2010) die Reduktion der Angst als angestrebtes Ziel fest, was auch aus den vorliegenden Ergebnissen hervorgeht.

\section{Stärken und Limitationen}

Die Stärken der Studie liegen in der Anwendung des IM und der Methodik der Datenerhebung. Vor allem ermöglichten die zwei Formen der Datenerhebung - Fokusgruppeninterviews und Einzelinterviews - zahlreichen Gesundheitsexpertinnen und -experten aus unterschiedlichen $\mathrm{Ge}$ sundheitsberufen sowie aus 3 Settings der Gesundheitsversorgung, an dieser Studie teilzunehmen. Dadurch können unterschiedliche Perspektiven in das pSPP einfließen, was zur Verallgemeinerung der Ergebnisse führt. Besonders erwähnenswert ist auch, dass die Teilnehmenden aus dem Pflegeberuf, welche in Zukunft das Programm leiten könnten, ihre fundierten Ansichten in die Ergebnisse einbrachten.
Die Studie weist einige Limitationen auf. Erstens konnten im Rahmen von Fokusgruppeninterviews die Teilnehmenden über die Gestaltung der Versorgung von Menschen nach ACS in der Übergangsphase offen diskutieren. Aber sie bezogen durch die Gruppenkonstellation ihre Sicht über das pSPP und die Rolle der CR nurse nur auf die bestehenden Rahmenbedingungen. Daher ist nicht auszuschließen, dass andere Vorstellungen über die Befugnisse der CR nurses und die Eingliederung der Anlaufstelle nicht frei geäuBert wurden. Zweitens berief sich ihre Sicht hauptsächlich auf Interventionen zum Umgang mit der Krankheit, nicht auf Interventionen zur Gesundheitsförderung. Die theoretischen Hintergründe der pflegerischen Intervention wurden von den Teilnehmenden nicht erwähnt. Dementsprechend konnten die Ergebnisse solche Ansichten nicht erfassen. Drittens wurden die Ergebnisse nicht von den Gesundheitsexpertinnen und -experten validiert. Daher besteht eine Einschränkung hinsichtlich der Vertrauenswürdigkeit der Ergebnisse.

\section{Schlussfolgerung}

Das zu entwickelnde Programm kann dazu dienen, die nichtmedikamentöse Sekundärprävention bei den Betroffenen im Akutkrankenhaus zu initiieren und nach der Entlassung fortzuführen. Als Rahmenbedingungen müssen eine Anlaufstelle für Patientinnen und Patienten sowie die Funktion einer CR nurse geschaffen werden. Somit kann diese spezialisierte Pflegeperson die Betroffenen in der Übergangsphase begleiten, während sie die Patientenedukation auf Basis des personenzentrierten Ansatzes durchführt.

Um die oben genannten Limitationen zu minimieren, werden weitere Forschungen für die Entwicklung des pSPP benötigt, wie z.B. eine systematische Literaturarbeit, um sowohl die effektive pflegerische Intervention zur Gesundheitsförderung als auch deren theoretischen Hintergrund darzustellen. Somit kann das zu entwickelnde pSPP auf Basis der unterschiedlichen Wissensquellen konzeptualisiert werden. Weitere Forschungen beziehen sich auf die Entwicklung des Implementierungsplans und die Testung der Effektivität des pSPP, um es implementieren zu können.

Danksagung Wir bedanken uns bei allen Gesundheitsexpertinnen und -experten für die Teilnahme an dieser Studie. Vielen Dank an Eva Paltinger-Armbruster für die sorgfältige Transkription und an Mag. ${ }^{a}$ Verena Hauser für das gewinnbringende Lektorat des Artikels. Weiter bedanken wir uns bei den Kolleginnen und Kollegen der Erstautorin für die konstruktiven Diskussionen im Forschungskolloquium.

Förderung Medizinisch-Wissenschaftlicher Fonds des Bürgermeisters der Bundeshauptstadt 
Author Contribution Beitrag zur Konzeption oder zum Design der Arbeit: H. Qin, H. Mayer, D. Bonderman; Beitrag bei der Erfassung, Analyse oder der Interpretation der Daten: H. Qin, H. Mayer, S. Brunner, T. Großbichler; Manuskripterstellung: H. Qin; Kritische Überarbeitung von wichtigen intellektuellen Inhalten des Manuskripts: H. Qin, D. Bonderman, S. Brunner, T. Großbichler, R. Badr Eslam, H. Mayer; Genehmigung der letzten Version des Manuskripts zur Publikation: H. Qin, D. Bonderman, S. Brunner, T. Großbichler, R. Badr Eslam, H. Mayer; Bereitschaft, für alle Aspekte der Arbeit Verantwortung zu übernehmen: H. Qin, D. Bonderman, S. Brunner, T. Großbichler, R. Badr Eslam, H. Mayer.

Funding Open access funding provided by University of Vienna.

Interessenkonflikt H. Qin, D. Bonderman, S. Brunner, T. Großbichler, R. Badr Eslam und H. Mayer geben an, dass kein Interessenkonflikt besteht.

Open Access Dieser Artikel wird unter der Creative Commons Namensnennung 4.0 International Lizenz veröffentlicht, welche die Nutzung, Vervielfältigung, Bearbeitung, Verbreitung und Wiedergabe in jeglichem Medium und Format erlaubt, sofern Sie den/die ursprünglichen Autor(en) und die Quelle ordnungsgemäß nennen, einen Link zur Creative Commons Lizenz beifügen und angeben, ob Änderungen vorgenommen wurden.

Die in diesem Artikel enthaltenen Bilder und sonstiges Drittmaterial unterliegen ebenfalls der genannten Creative Commons Lizenz, sofern sich aus der Abbildungslegende nichts anderes ergibt. Sofern das betreffende Material nicht unter der genannten Creative Commons Lizenz steht und die betreffende Handlung nicht nach gesetzlichen Vorschriften erlaubt ist, ist für die oben aufgeführten Weiterverwendungen des Materials die Einwilligung des jeweiligen Rechteinhabers einzuholen.

Weitere Details zur Lizenz entnehmen Sie bitte der Lizenzinformation auf http://creativecommons.org/licenses/by/4.0/deed.de.

\section{Literatur}

Alsen P, Brink E, Persso LO, Brandstrom Y, Karlson BW (2010) Illness perceptions after myocardial infarction: relations to fatigue, emotional distress, and health-related quality of life. J Cardiovasc Nurs 25(2):E1-E10. https://doi.org/10.1097/JCN. 0b013e3181c6dcfd

Auer R, Gaume J, Rodondi N, Cornuz J, Ghali WA (2008) Efficacy of in-hospital multidimensional interventions of secondary prevention after acute coronary syndrome: a systematic review and meta-analysis. Circulation 117(24):3109-3117. https://doi.org/ 10.1161/CIRCULATIONAHA.107.748095

Bartholomew Eldredge LK, Markham CM, Ruiter RAC, Kok G, Parcel GS (2016) Planning health promotion programs: an intervention mapping approach, 4. Aufl. John Wiley \& Sons, San Francisco

Berra K (2010) Challenges of changing lifestyle to reduce risk for cardiovascular disease. J Cardiovasc Nurs 25(3):223-227. https:// doi.org/10.1097/JCN.0b013e3181cec7e4

Biga C (2021) The continuum of care: from inpatient and outpatient cardiac rehabilitation to long-term secondary prevention. In: American Association of Cardiovascular and Pulmonary Rehabilitation (Hrsg) Guidelines for cardiac rehabilitation programs, 6. Aufl. Human Kinetics, Champain, S 7-13

BMGF (2016) Verbesserung der Gesprächsqualität in der Krankenversorgung. https://oepgk.at/wp-content/uploads/2018/10/strategiezur-verbesserung-der-gespraechsqualitaet.pdf. Zugegriffen: 14. Febr. 2021 (Strategie zur Etablierung einer patientenzentrierten Kommunikationskultur. Beschlossen von der Bundeszielsteuerungskommission am 1. Juli 2016)
Braun V, Clarke V (2006) Using thematic analysis in psychology. Qual Res Psychol 3(2):77-101. https://doi.org/10.1191/ 1478088706qp063oa

Clark AM, King-Shier KM, Duncan A, Spaling M, Stone JA, Jaglal S, Angus J (2013) Factors influencing referral to cardiac rehabilitation and secondary prevention programs: a systematic review. Eur J Prev Cardiolog 20(4):692-700. https://doi.org/10.1177/ 2047487312447846

Collet J-P, Thiele H, Barbato E et al (2020) ESC Guidelines for the management of acute coronary syndromes in patients presenting without persistent ST-segment elevation. Eur Heart J. https://doi. org/10.1093/eurheartj/ehaa575

Cossette S, Frasure-Smith N, Dupuis J, Juneau M, Guertin MC (2012) Randomized controlled trial of tailored nursing interventions to improve cardiac rehabilitation enrollment. Nurs Res 61(2):111-120. https://doi.org/10.1097/NNR.0b013e318240dc6b

Craig P, Dieppe P, Macintyre S, Michie S, Nazareth I, Petticrew M (2013) Developing and evaluating complex interventions: the new medical research council guidance. Int J Nurs Stud 50(5):587-592. https://doi.org/10.1016/j.ijnurstu.2012.09.010

Dalal HM, Doherty P, Taylor RS (2015) Cardiac rehabilitation. BMJ 351:h5000. https://doi.org/10.1136/bmj.h5000

Flodgren G, Eccles MP, Shepperd S, Scott A, Parmelli E, Beyer FR (2011) An overview of reviews evaluating the effectiveness of financial incentives in changing healthcare professional behaviours and patient outcomes. Cochrane Database Syst Rev. https://doi. org/10.1002/14651858.CD009255

Froschauer U, Lueger M (2020) Das qualitative Interview: Zur Praxis interpretativer Analyse sozialer Systeme, 2. Aufl. UTB, Wien

Großschädl F, Burkert N, Muckenhuber J, Rásky É, Freidl W (2014) Myokardinfarkt: Medizinische Versorgungskette und Gesundheitskompetenz. https://www.sozialversicherung.at/cdscontent/ load ?contentid $=10008.714830 \&$ version $=1420619129$. Zugegriffen: 16. Dez. 2020

Hambraeus K, Tydén P, Lindahl B (2016) Time trends and gender differences in prevention guideline adherence and outcome after myocardial infarction: Data from the SWEDEHEART registry. Eur J Prev Cardiolog 23(4):340-348. https://doi.org/10.1177/ 2047487315585293

Huriani E (2019) Myocardial infarction patients' learning needs: perceptions of patients, family members and nurses. Int J Nurs Sci 6(3):294-299. https://doi.org/10.1016/j.ijnss.2019.05.001

Kotseva K, Wood D, De Backer G, De Bacquer D (2013) Use and effects of cardiac rehabilitation in patients with coronary heart disease: results from the EUROASPIRE III survey. Eur J Prev Cardiol 20(5):817-826. https://doi.org/10.1177/2047487312449591

Mayer H (2019) Pflegeforschung anwenden: Elemente und Basiswissen für das Studium, 5. Aufl. Facultas, Wien

McCance T, McCormack B (2017) The person-centred practice framework. In: McCormack B, McCance T (Hrsg) Person-centred practice in nursing and health care: theory and practice, 2 . Aufl. John Wiley \& Sons, Oxford, S 36-64

Meuser M, Nagel U (2009) Das Experteninterview - konzeptionelle Grundlagen und methodische Anlage. In: Pickel S, Pickel G, Lauth H, Jahn D (Hrsg) Methoden der vergleichenden Politikund Sozialwissenschaft - Neue Entwickleunhen und Anwendungen. VS, Wiesbaden, S 465-479 https://doi.org/10.1007/978-3531-91826-6_23

Mülhauser S, Bonhôte Börner M, Saner H, Zumstein-Shaha M (2018) Auswirkung motivierender Gesprächsführung auf die Krankheitswahrnehmung bei stabiler koronarer Herzkrankheit. Pflege 31(2):75-85. https://doi.org/10.1024/1012-5302/a000595

Murphy B, Le Grande M, Alvarenga M, Worcester M, Jackson A (2019) Anxiety and depression after a cardiac event: prevalence and predictors. Front Psychol. https://doi.org/10.3389/fpsyg. 2019.03010

Oranta O, Luutonen S, Salokangas RKR, Vahlberg T, Leino-Kilpi H (2010) The outcomes of interpersonal counselling on depressive 
symptoms and distress after myocardial infarction. Nord J Psychiatry 64(2):78-86. https://doi.org/10.3109/08039480903402227

Patientencharta (2006) Vereinbarung zur Sicherstellung der Patientenrechte (Patietencharta). https://www.ris.bka.gv.at/GeltendeFassung. wxe? Abfrage $=$ Bundesnormen $\&$ Gesetzesnummer $=20004633 \&$ FassungVom=2021-02-14. Zugegriffen: 14. Febr. 2021

Piepoli MF, Hoes AW, Agewall S et al (2016) 2016 European Guidelines on cardiovascular disease prevention in clinical practice. Eur Heart J 37(29):2315-2381. https://doi.org/10.1093/eurheartj/ ehw106

Pocock SJ, Brieger D, Gregson J et al (2020) Predicting risk of cardiovascular events 1 to 3 years post-myocardial infarction using a global registry. Clin Cardiol 43(1):24-32. https://doi.org/10. $1002 /$ clc. 23283

Schnurr S (2003) Vignetten in quantitativen und qualitativen Forschungsdesigns. In: Otto H-U, Oelerich G, Micheel H-G (Hrsg) Empirische Forschung und Soziale Arbeit. Luchterhand, Neuwied, S 393-400

Schrems B (2019) Vulnerabilität in der professionellen Pflegebeziehung. Eine Synopsis zum Vortrag am 6. November $2019 \mathrm{im}$ Rahmen des 4. Pflege Professionell Symposiums - Zeit für Pflege. Pflege Professionell 24/2019, 01-04. https://pflege-professionell. at/vulnerabilitaet-in-der-professionellen-pflegebeziehung. Zugegriffen: 16.01.2021

Snaterse M, Dobber J, Jepma P et al (2016) Effective components of nurse-coordinated care to prevent recurrent coronary events: a systematic review and meta-analysis. Heart 102(1):50. https:// doi.org/10.1136/heartjnl-2015-308050

Statistik Austria (2019) Gestorbene in Österreich ab 1970 nach Todesursachen und Geschlecht. https://www.statistik.at/web_de/ statistiken/menschen_und_gesellschaft/gesundheit/todesursachen/ index.html. Zugegriffen: 16. Dez. 2020

Sulzgruber P, Gall W, Niessner A et al (2019) Projekt „QI-KA“ - Sektorübergreifende kardiologische Qualitätsindikatoren für das österreichische Gesundheitswesen. https://www.sozialversicherung. at/cdscontent/load?contentid $=10008.738744 \&$ version $=1599468203$. Zugegriffen: 16. Dez. 2020

The Joanna Briggs Institute (2010) Best practice information sheet: the Joanna Briggs institute best practice information sheet. Nurs Health Sci 12(3):288-291. https://doi.org/10.1111/j.1442-2018. 2010.00548.x

WHO (2020) The top 10 causes of death fact sheet. http://www.who. int/mediacentre/factsheets/fs310/en/. Zugegriffen: 16. Dez. 2020

Wienbergen H, Fach A, Meyer S et al (2018) Effects of an intensive long-term prevention programme after myocardial infarction. Eur J Prev Cardiolog 0(00):1-9. https://doi.org/10.1177/ 2047487318781109

Hinweis des Verlags Der Verlag bleibt in Hinblick auf geografische Zuordnungen und Gebietsbezeichnungen in veröffentlichten Karten und Institutsadressen neutral. 